\title{
Asertividad en el diagnóstico clínico de los tumores cutáneos
}

\author{
Viviana Zemelman ${ }^{1 a}$, Carlos Y Valenzuela ${ }^{2}$, Felix Fisch ${ }^{3}$, \\ Johanna Road ${ }^{4}$, Juan H oneyman ${ }^{1}$.$$
\begin{aligned}
& \text { Assessment of clinical diagnostic } \\
& \text { accuracy for skin cancer }
\end{aligned}
$$

Background: There is an increase in the incidence rates of skin cancer in Chile. Aim: To study the clinical diagnostic accuracy (CDA) for skin cancer. Ma terial y methods: CDA was defined as the percentage of agreement between clinical and pathological diagnosis. Approximately 600,000 pathological reports from five hospitals in Santiago were reviewed. A total of 2,593 skin tumours; 230 Malignant Melanoma (MM); 412 Squamous Cell Carcinoma (SCC) and 1,951 Basal Cell Carcinoma (BCC) were studied. These tumours were clinically diagnosed and surgically treated by dermatologists. The CDA was studied for each tumour, by the anatomical localization of the tumour, Breslow Index in MM, by age and sex of the patient. Results: The highest CDA was observed for BCC (76.2\%); followed by MM (64.3\%) and SCC (34.7\%). By anatomical localization, for MM the highest CDA was observed in the soles ( $p$ $<0.05)$; for BCC, the hightest CDA was in the face $(\mathrm{p}<0.05)$. No significant differences were observed in SCC. By age, for MM, the CDA was higher in patients aged less than 50 years. No differences in CDA by age were observed in the other two tumours. By sex, no differences were found. A higher CDA was found in MM with Breslow indexes III and IV than for MM with Breslow indexes I and II. Conclusions: CDA is affected by the clinical variables analyzed in this study. A more accurate clinical diagnosis of skin cancer could be obtained taking into account these variables (Rev Méd Chile 2003; 131: 1421-7).

(Key Words: Melanoma; Skin neoplasms)

Recibido el 23 de mayo, 2003. Aceptado el 16 de septiembre, 2003.

${ }^{1}$ Departamento de Dermatología, Hospital Clínico de la Universidad de Chile; ${ }^{2}$ Programa Genética Humana, ICBM, Facultad de Medicina, Universidad de Chile; ${ }^{3}$ Servicio de Dermatología, Hospital San Borja - Arriarán; ${ }^{4}$ Escuela de Postgrado; Facultad de Medicina, Universidad de Chile.

aBioquímico.

$\mathrm{E}^{\mathrm{n}}$ las últimas dos décadas, se ha observado un incremento de la incidencia de cáncer de piel en el ámbito mundial ${ }^{1-4}$. Este incremento es particularmente importante en el melanoma, que ha duplicado su incidencia mundial por cada

Correspondencia a: Viviana Zemelman. Casilla 249-11, Ñuña, Santiago, Chile. Fono: 2234771.

E mail: vzemelma@ns.hospital.uchile.cl década ${ }^{5}$. En Chile, se ha observado un aumento de la mortalidad por melanoma maligno $(\mathrm{MM})^{6}$. También, se ha informado un incremento en las tasas de incidencia de cáncer cutáneo en los hospitales públicos de la Región Metropolitana7. El aumento de la mortalidad y morbilidad de cáncer de piel ha motivado estudiar las variables que intervienen en su diagnóstico clínico e histopatológico. Un diagnóstico preciso y precoz de 
cáncer cutáneo podría reducir su mortalidad, el sufrimiento de los pacientes y los costos de la enfermedad. En la literatura internacional, existen varias publicaciones sobre el grado de precisión del diagnóstico clínico del cáncer de piel, sobre todo en el caso del MM, donde se ha investigado el número de casos falsos negativos y los casos falsos positivos. En un estudio realizado en Escocia, se diagnosticaron 163 MM clínicamente, de los cuales, 113 fueron confirmados histológicamente como MM. En este mismo estudio, se confirmó que el acierto diagnóstico de un dermatólogo de más de 10 años de experiencia es $80 \%$, en cambio para aquellos dermatólogos de sólo 3-5 años, este acierto disminuye a $62 \%^{8}$. Por otra parte, en un estudio realizado en Estados Unidos de Norteamérica, donde se examinaron 1.946 informes histopatológicos de biopsias enviadas como nevus (lesiones benignas), se encontró que 2,3\% de ellas correspondían a lesiones malignas (12 MM, 30 cáncer basocelular (CBC), 3 cáncer espinocelular $(\mathrm{CEC}))^{9}$. En otro análisis, donde se revisó la base de datos computarizada (13.878 lesiones), se encontraron 214 lesiones clínica e histológicamente diagnosticadas como MM, 79 lesiones cutáneas diagnosticadas clínicamente como MM no fueron confirmadas histológicamente como tales; en este mismo estudio, el diagnóstico clínico de MM fue realizado en $84,5 \%$ de los casos de MM confirmados histológicamente ${ }^{10}$.

El aumento de la mortalidad y de la morbilidad del cáncer cutáneo en Chile, representa un problema importante de salud pública. En este contexto, es interesante estudiar parámetros del diagnóstico clínico de estos tumores. Este estudio tiene por objetivo principal, estudiar el índice de asertividad en el diagnóstico clínico de tumores cutáneos por dermatólogos de hospitales públicos de Santiago y analizar variables de importancia que influyen en dicho índice.

\section{Material y MÉTOdos}

Se analizaron retrospectivamente aproximadamente 600.000 informes histopatológicos de biopsias realizadas en el período comprendido entre los años 1992 y 2001, pertenecientes a 5 hospitales públicos de la Región Metropolitana (hospitales del Salvador, Barros Luco-Trudeau, San Juan de Dios, San Borja-Arriarán y Sótero del Río). Estos hospitales cubren una población aproximada de 2.500.000 habitantes, cerca de la mitad de la población de Santiago y representa aproximadamente $17 \%$ de la población chilena. Se registraron 5.750 tumores cutáneos en los cuales se analizó: edad y sexo de los pacientes, localización anatómica, tipo clínico e histológico del tumor e índice de Breslow en los MM. Del total de 5.750 tumores cutáneos registrados para este estudio, 2.593 (45\%) tumores fueron biopsiados y enviados directamente a examen histopatológico por dermatólogos. El 55\% restante fue enviado por otros especialistas como cirujanos, oncólogos, médicos internistas, etc. Debe considerarse que este 55\% incluye aquellos tumores cutáneos diagnosticados clínicamente como tales por dermatólogos y referidos a otros especialistas. Cabe señalar que en el informe histopatológico, sólo es registrado el servicio de procedencia de la biopsia. No se analizó la información proveniente de servicios distintos a dermatología, porque una proporción de los tumores de estos servicios proviene con diagnóstico clínico de otros servicios incluidos los de dermatología. El índice de asertividad (IA) en el diagnóstico clínico de estos tumores se analizó por tipo de tumor, por localización anatómica del tumor, tipo histológico, por índice de Breslow en el MM y por edad y sexo del paciente. Debemos notar que nuestro estudio es retrospectivo, recoge como dato inicial el diagnóstico histopatológico de tumores cutáneos, se registran todos los casos de MM, CEC y CBC, se analizan los diagnósticos clínicos diferenciales previa biopsia para cada tumor. A la coincidencia entre el diagnóstico histopatológico y el clínico llamaremos índice de asertividad (IA).

Análisis estadístico. Se realizó el test de Chi cuadrado y una prueba $\mathrm{z}$ de proporciones para calcular la significación estadística. Los valores de $\mathrm{p}<0,05$ fueron considerados significativos.

\section{Resultados}

De un total de $658 \mathrm{MM}$ diagnosticados histológicamente, $230 \mathrm{MM}$ (35\%) fueron biopsiados y envia- 
dos directamente por dermatólogos; de los que $64,3 \%$ fue diagnosticado clínicamente como MM. El 35,7\% restante fue enviado con otros diagnósticos entre los cuales destacaron: $10,4 \%$ como nevo pigmentario, 6,2\% como $\mathrm{CBC}, 7,8 \%$ no tenía consignado el diagnóstico clínico en el informe histopatológico (Tabla 1). De un total de 1.150 CEC diagnosticados histológicamente 412 (36\%) fueron biopsiados y enviados directamente por dermatólogos a examen histológico, de los cuales $36,2 \%$ fue diagnosticado clínicamente como CEC. El 63,8\% restante llegó con otro diagnóstico clínico, dentro de los que se destacó el de CBC $(23,1 \%)$ (Tabla 1). De un total de 3.942 CBC diagnosticados histológicamente, 1.951 (49\%) fueron diagnosticados y enviados a examen histológico por dermatólogos de los que $77,5 \%$ fue diagnosticado clínicamente como CBC, el 22,5\%

Tabla 1. D iagnósticos clínicos diferenciales para cada tumor

\begin{tabular}{|c|c|c|c|c|c|c|}
\hline \multirow[t]{2}{*}{ Diagnóstico clínico* } & \multicolumn{2}{|c|}{$\mathrm{MM}^{* *}$} & \multicolumn{2}{|c|}{ CEC** } & \multicolumn{2}{|c|}{$\mathrm{CBC}^{* *}$} \\
\hline & $\mathrm{N}^{\circ}$ & $\%$ & $\mathrm{~N}^{\circ}$ & $\%$ & $\mathrm{~N}^{\circ}$ & $\%$ \\
\hline Acrocordon & 0 & & 0 & & 3 & 0,2 \\
\hline Angioma & 1 & 0,4 & 0 & & 5 & 0,2 \\
\hline Cáncer & 0 & & 8 & 1,9 & 4 & 0,2 \\
\hline Carcinoma basocelular & 14 & 6,2 & 95 & 23,1 & 1.511 & 77,5 \\
\hline Carcinoma espinocelular & 1 & 0,4 & 149 & 36,2 & 37 & 2,0 \\
\hline Cuerno cutáneo & 0 & & 8 & 1,9 & 0 & \\
\hline Dermatofibroma & 1 & 0,4 & 0 & & 0 & \\
\hline Enfermedad de Bowen & 1 & 0,4 & 6 & 1,5 & 19 & 1,0 \\
\hline Fibroma & 0 & & 0 & & 4 & 0,2 \\
\hline Granuloma & 0 & & 8 & 1,9 & 0 & \\
\hline Granuloma piógeno & 2 & 0,8 & 0 & & 3 & 0,2 \\
\hline Hidrocistoma apocrino & 0 & & 0 & & 3 & 0,2 \\
\hline Hiperplasia sebacea & 0 & & 0 & & 2 & 0,1 \\
\hline Lesión & 0 & & 0 & & 30 & 1,5 \\
\hline Lesión pigmentada & 7 & 3,0 & 0 & & 0 & \\
\hline Melanoma maligno & 148 & 64,3 & 1 & 0,2 & 28 & 1,4 \\
\hline Nevo pigmentado & 24 & 10,4 & 0 & & 0 & \\
\hline Nevus atípico & 3 & 1,4 & 0 & & 22 & 1,1 \\
\hline Nevus tuberoso & 0 & & 0 & & 7 & 0,3 \\
\hline Nevus & 0 & & 0 & & 30 & 1,5 \\
\hline Nódulo & 0 & & 0 & & 5 & 0,2 \\
\hline Papiloma & 0 & & 0 & & 5 & 0,2 \\
\hline Queratoacantoma & 0 & & 23 & 5,6 & 8 & 0,4 \\
\hline Queratosis & 0 & & 17 & 4,1 & 0 & \\
\hline Queratosis actínica & 1 & 0,4 & 17 & 4,1 & 13 & 0,7 \\
\hline Queratosis seborreica & 6 & 2,7 & 16 & 4,0 & 24 & 1,2 \\
\hline Quiste & 0 & & 0 & & 8 & 0,4 \\
\hline Tumor & 3 & 1,4 & 17 & 4,1 & 34 & 1,7 \\
\hline Ulcera & 0 & & 1 & 0,2 & 2 & 0,1 \\
\hline Verruga & 0 & & 1 & 0,2 & 0 & \\
\hline No consignado & 18 & 7,8 & 45 & 11,0 & 144 & 7,5 \\
\hline Total & 230 & 100,0 & 412 & 100,0 & 1.951 & 100,0 \\
\hline
\end{tabular}

MM: Melanoma maligno, CEC: Carcinoma espinocelular, CBC: Carcinoma basocelular. Diagnóstico clínico*: Diagnóstico clínico realizado por el dermatólogo que aparece en el informe histopatológico **= Diagnóstico histopatológico. 
restante presentó distintos diagnósticos (Tabla 1). Se observa en la Tabla 1 que 23,1\% de los CEC histológicos fueron diagnosticados clínicamente como CBC; sin embargo, sólo $2 \%$ de los CBC histológicos fue diagnosticado como CEC clínicamente. También, se observó que 6,2\% del total de los MM histológicamente confirmados, fueron diagnosticados clínicamente como CBC, en cambio, solo 1,4\% de CBC histológicos fueron diagnosticados como MM clínicamente. Se observa, también, en la Tabla 1, que el tumor de mayor correlación clínico-histopatológica es el CBC (77,5\%), seguido del MM (64,3\%), y del CEC $(36,2 \%)$.
Con relación a la ubicación anatómica del tumor, cuando se comparó cara con zonas no fotoexpuestas, el CBC presentó un mayor índice de asertividad en cara que en zonas no fotoexpuestas ( $p<0,05)$. Esto no se observó en los otros dos tumores (Figura 1).

Cuando se analizaron las principales localizaciones de estos tumores, en el caso del MM, el mayor IA se observó en los MM plantares (94,4\%) $(\mathrm{p}<0,05)$ (Figura 2). Cabe señalar que del total de MM derivados por dermatólogos el mayor porcentaje correspondió a los encontrados en cara $(40,2 \%)$ seguido de piernas (21,3\%), tronco $(15,2 \%)$ y planta $(11,0 \%)$. En el caso del CBC, la

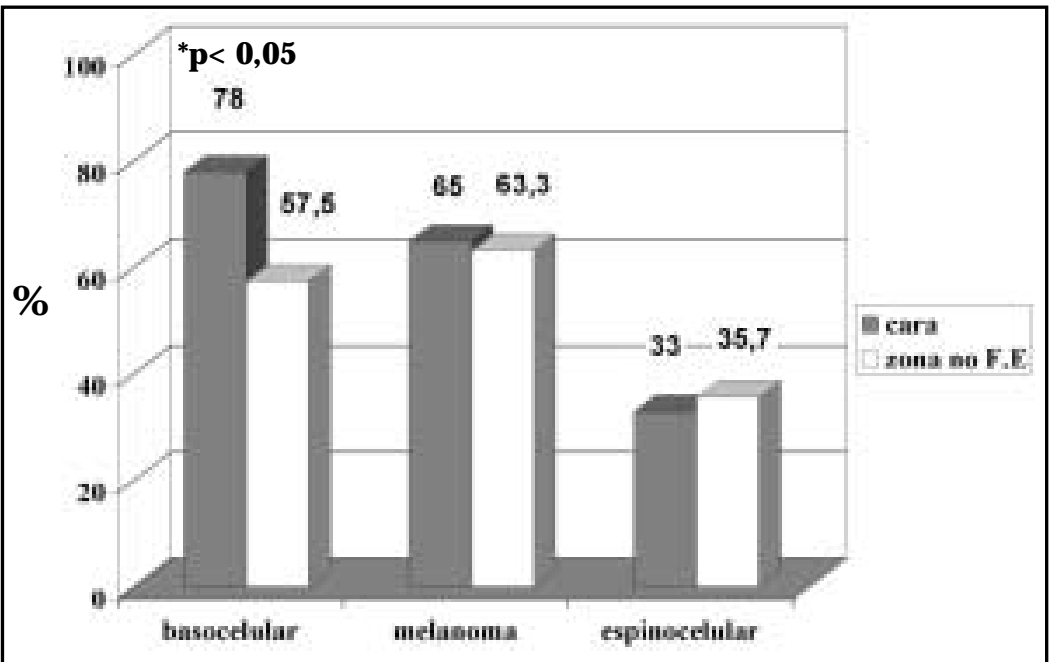

FiguRa 1. Índice de asertividad por localización según fotoexposición.

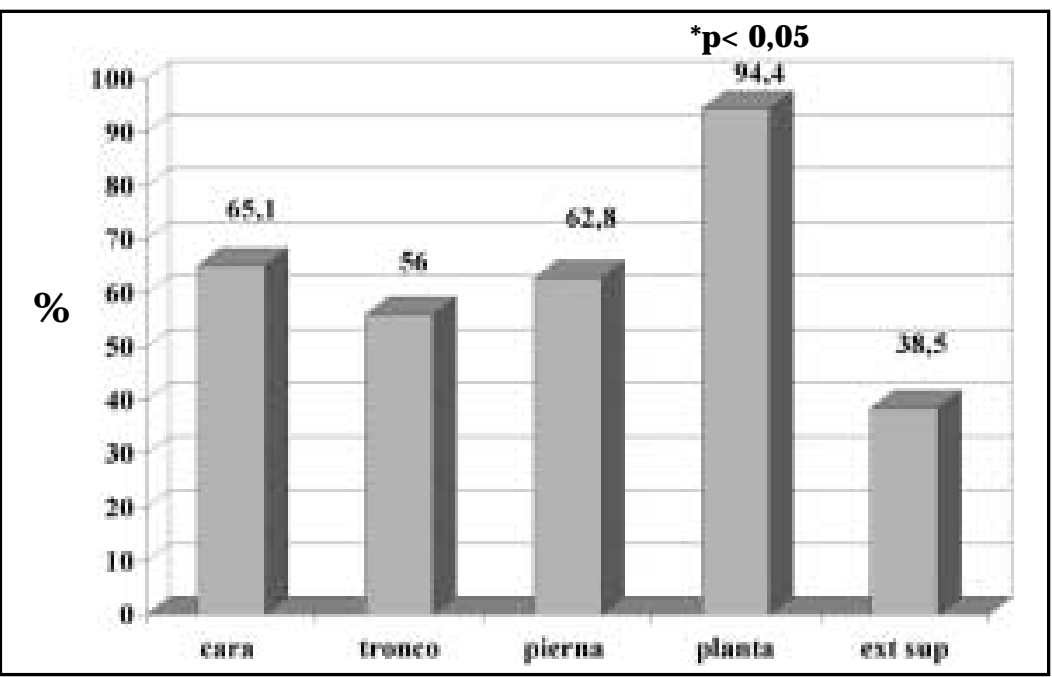

FIgURA 2. Índice de asertividad por localización en melanoma maligno. 
cara presentó el mayor IA $(78 \%)(p<0,05)$ (Figura 3). El mayor número de CBC derivados por dermatólogos correspondió a los de cara (87,8\%), seguido de tórax $(8,8 \%)$ y extremidades superiores $(1,7 \%)$. En el CEC, no se encontró diferencias significativas en el IA entre las principales localizaciones (Figura 4). Las localizaciones de CEC fueron cara $(77,8 \%)$, piernas $(8,6 \%)$, tórax $(6,7 \%)$ y zona genital $(2,9 \%)$.

Según edad, se observó un mayor IA en MM en los pacientes menores de 50 años (Figura 5). Estos resultados no fueron observados en $\mathrm{CBC}$ ni en CEC. Es necesario destacar que no se encontraron diferencias significativas en cuanto al IA según sexo en ninguno de los tumores.
Al estudiar el índice de asertividad de los MM por índice de Breslow, se observó una mayor asertividad en aquellos MM con índice Breslow III y IV que en los con índice I y II. Los porcentajes de IA para los grados I, II, III y IV fueron $56,5 \%$, $62,0 \%, 73,8 \%$ y $69,5 \%$, respectivamente. Este porcentaje para las categorías I+II y III+IV fue $57,9 \%$ y $72,3 \%$ que difieren significativamente con la prueba $\mathrm{z}$ unilateral de proporciones $(\mathrm{p}=0,024)$.

\section{Discusión}

Al analizar el diagnóstico clínico con que se enviaron las muestras tumorales, una vez sabido
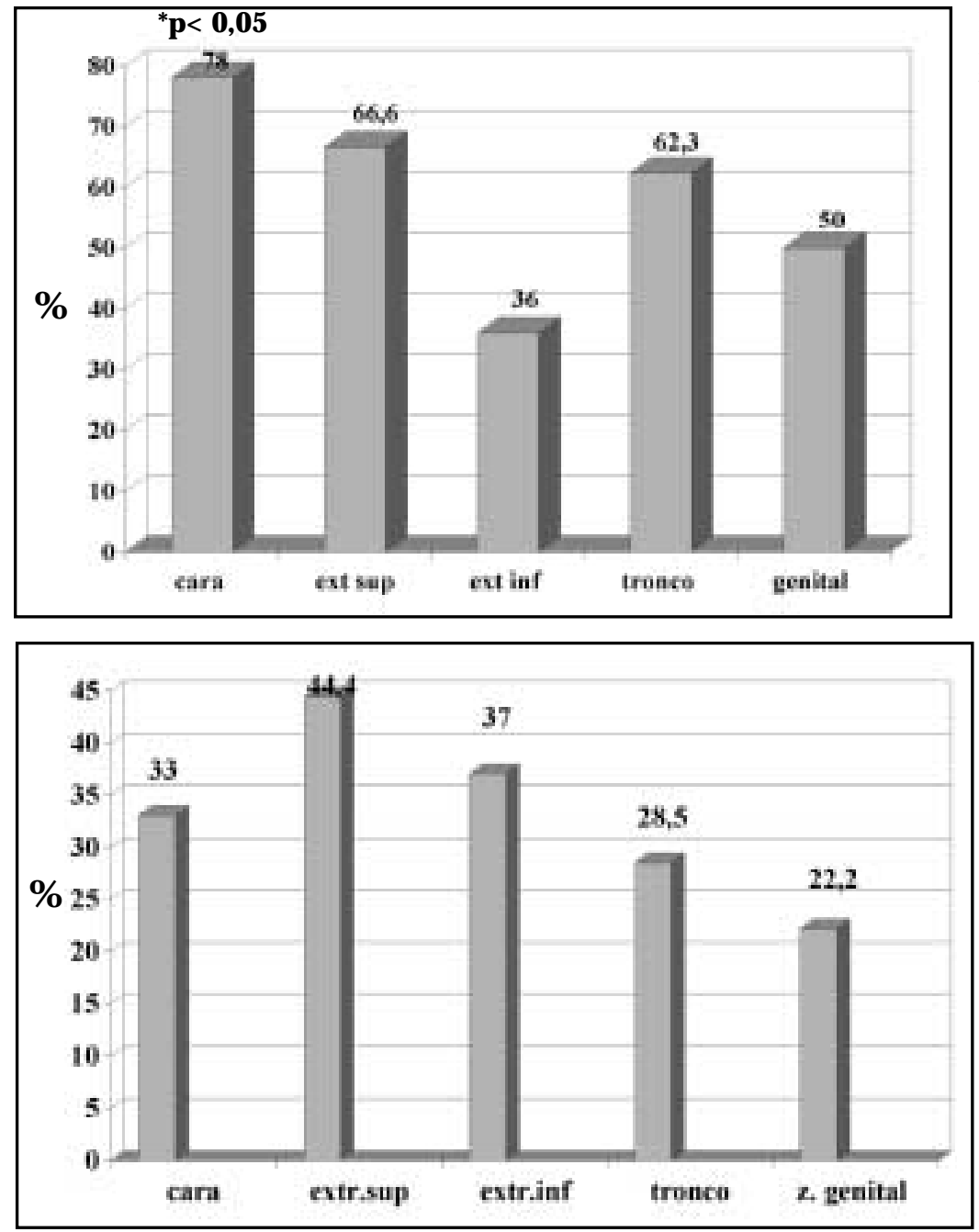

Figura 3. Índice de asertividad por localización en carcinoma basocelular.

FiguRa 4. Índice de asertividad por localización en carcinoma espinocelular. 


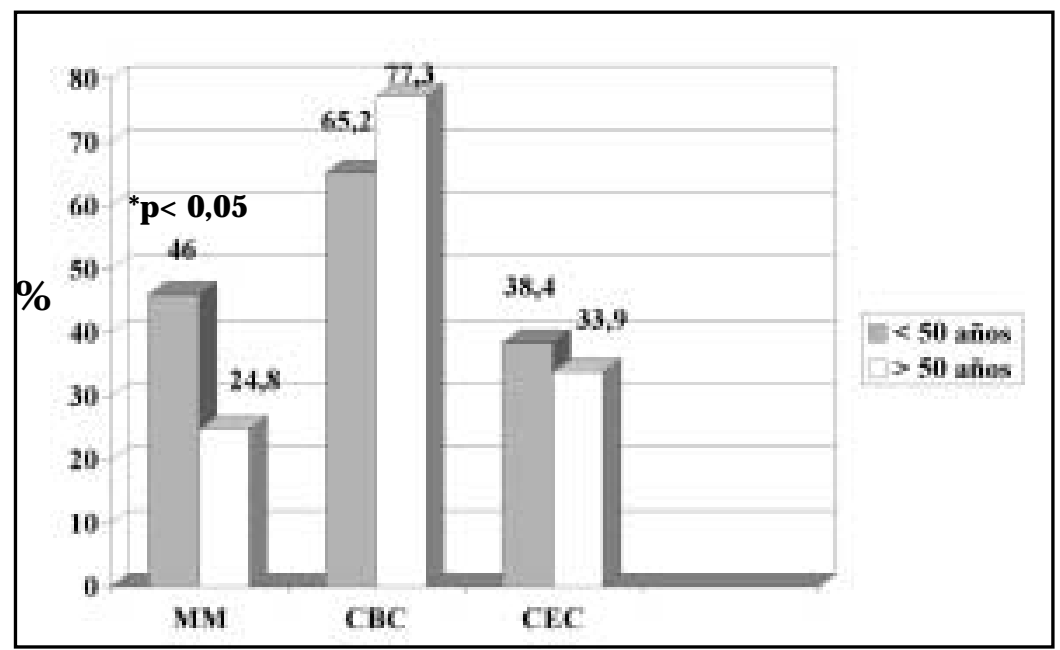

FIguRA 5. Índice de asertividad según edad.

el diagnóstico histopatológico, llamó la atención que el correlato clínico-histológico fue en orden decreciente para CBC (77,5\%), MM (64,3\%) y CEC (36,2\%). Esta diferencia podría explicarse porque CEC se presenta clínicamente simulando otras patologías en mayor proporción que los otros tumores. Entre esas patologías destacan CBC $(23,1 \%)$, queratoacantoma $(5,6 \%)$, queratosis actínica $(4,1 \%)$, queratosis $(4,1 \%)$ y tumor inespecífico de piel (4,1\%). Aunque MM presenta un alto IA en nuestro estudio, un gran porcentaje de los MM histológicos ingresó como nevo pigmentado $(10,4 \%)$. Estos nevos son aquellos en que el dermatólogo ha sospechado necesario su estudio histológico; lo que reafirma la recomendación que cuando un nevo pigmentado cambia su comportamiento o se asocian a él otros signos debe ser examinado por un especialista. Es interesante destacar que en el CEC, 23,1\% fueron diagnosticados clínicamente como $\mathrm{CBC}$, lo que no sucede a la inversa, sólo $2 \%$ de los $\mathrm{CBC}$ histológicos fueron diagnosticados clínicamente como CEC. Esto podría deberse a que el $\mathrm{CBC}$ es un tumor mucho más frecuente que el $\mathrm{CEC}$, lo que lleva al dermatólogo a pensar en $\mathrm{CBC}$ con mayor frecuencia. El diagnóstico clínico en este caso estaría influido por un factor frecuencia. El MM presenta una situación intermedia, esto se debería a que el MM tiene un patrón clínico más definido que el CEC, 6,2\% de los MM histológicos fueron clínicamente diagnosticados como CBC, y casi ninguno como CEC $(0,4 \%)$, esto podría deberse a que el
CBC pigmentado puede confundirse clínicamente con MM.

El 64,3\% de IA en el MM observado en nuestro trabajo es similar a lo informado por un estudio realizado en Suecia, donde se estudiaron 174 MM confirmados microscópicamente. De estos MM, 65,5\% había sido diagnosticado clínicamente como $\mathrm{MM}^{11}$. La similitud encontrada entre este trabajo realizado en Suecia y lo observado en nuestro trabajo, donde el diagnóstico clínico procede de dermatólogos de hospitales públicos, es importante porque indica un nivel de diagnóstico clínico de MM comparable entre los dos países. Sin embargo, un estudio de EEUU informa que de 265 MM confirmados histológicamente, 80,7\% fue diagnosticado clínicamente, desde una base de datos de la Sección de Oncología. Creemos que esto puede explicarse por el sesgo debido a la gravedad de los tumores que llegan a oncología, como a las múltiples instancias diagnósticas previas $^{12}$. Asimismo, un estudio realizado en el Reino Unido presenta un alto porcentaje $(88,3 \%)$ de IA para $\mathrm{MM}^{13}$; este estudio proviene de un Departamento de Dermatología altamente especializado en melanoma y que podría ser un centro de referencia para MM.

La observación de un mayor IA en cara que en zonas no fotoexpuestas sólo en el carcinoma basocelular, puede explicarse porque este tumor se da preferencialmente en cara, entonces la sospecha de CBC facial tiene una mayor probabilidad de corresponder histológicamente a CBC. 
La magnitud del IA según localización anatómica fue muy distinta en los tres tumores. Llamó la atención el bajo índice de asertividad observado en CEC especialmente en zona genital, lo que podría explicarse porque si el CEC proviene de un condiloma acuminado malignizado, el diagnóstico clínico es muy difícil y con frecuencia este diagnóstico es realizado histológicamente. De todos modos, el CEC tiene muchos patrones clínicos, su diagnóstico es difícil, puede presentarse desde una pequeña lesión verrucosa hasta un gran tumor ulcerado. El mayor IA en MM se obtuvo para la localización plantar (94,4\%); lo que puede explicarse porque cuando se observa un nevo pigmentado plantar se agudiza su examen clínico, ya que tiene una alta probabilidad de ser MM.

La edad fue discriminativa en el caso de MM donde el IA fue mucho mayor en los pacientes menores de 50 años. Esto podría explicarse porque, en los mayores de 50 años, la frecuencia de los otros tumores es mayor y compiten por el

\section{REFERENCIAS}

1. Karagas MR, Greenberg ER, Spencer SK, Stukel tA, Mотт LA. Increase in incidence rates of basal cell and squamous cell skin cancer in New Hampshire, USA. New Hampshire Skin Cancer Study Group. Int J Cancer 1999; 81: 555-9.

2. URBACH F. Incidence of nonmelanoma skin cancer. Dermatol Clin 1991; 9: 751-5.

3. Gloster HM JR, Brodiand DG. The epidemiology of skin cancer. Dermatol Surg 1996; 22: 217-26.

4. Piesko I, Severi G, Obsitnikova A, Boyle P. Trends in the incidence of nonmelanoma skin cancer in Slovakia, 1978-1995. Neoplasma 2000; 47: 137-42.

5. Hughes BR, Altman DG, Newton JA. Melanoma and skin cancer: evaluation of a health education programme for secondary schools. Br J Dermatol 1993; 128: 412-7.

6. Zemelman V, Garmendia ML, Kirschbaum A. Malignant Melanoma mortality rates in Chile (1988-98). In J Dermatol 2002; 41: 99-103.

7. Zemelman V, Roa J, Díaz C, Araya I, Zamalloa G, FAÚNDEZ E. Aumento de la incidencia del cáncer diagnóstico clínico con MM en mayor proporción. Además, existen menos lesiones pigmentarias benignas en jóvenes que en mayores de 50 años, lo que favorece un diagnóstico más acertado de MM en menores de 50 años.

Con relación al índice de Breslow, el mayor IA observado en los MM Breslow III y IV que en los Breslow I y II parece explicarse porque la profundidad del tumor hace más obvio el diagnóstico clínico de MM.

Este estudio del índice de asertividad, nos mostró un buen acierto diagnóstico en carcinoma basocelular y en el melanoma maligno y un acierto moderado en carcinoma espinocelular. Por lo que, nuestro análisis revela que este tipo de estudios es adecuado para evaluar la apreciación clínica de los tumores cutáneos, ya que el diagnóstico clínico de cada tumor parece estar influido por las variables estudiadas. Un análisis más acucioso de este índice, debería conducir a un mejor diagnóstico clínico de esta neoplasia.

cutáneo en los hospitales públicos de la Región Metropolitana (1992-1998). Rev Dermatol (Chile) 2001; 17: 180-5.

8. Morton CA, Mackie RM. Clinical accuracy of the diagnosis of cutaneous malignant Melanoma. Br J Dermatol 1998; 138: 283-7.

9. Reeck MC, Chuang TY, Eads TJ, Faust HB, Farmer ER, Hood AF. The diagnostic yield in submitting nevi for histologic examination. J Am Acad Dermatol 1999; 40: 567-71.

10. Grin CM, Kopf AW, WeiKovich B, Barts RS, Levenstein MJ. Accuracy in the clinical diagnosis of malignant melanoma. Arch Dermatol 1990; 126: 763-6.

11. Weider F, Uebel G, Huben H. Diagnostic accuracy in epithelial skin tumours. Hautarzt 1981; 32: 359-63.

12. HoFer T. Malignant skin tumours in private dermatology practice. A quality control Study. Hautarzt 2002; 33: 666-71.

13. HАLоск GG, Lutz DA. Prospective study of the accuracy of the surgeon's diagnosis in 2000 excised skin tumours. Plast Reconstr Surg 1998; 10: $1255-61$. 